\title{
Clinical Factors Associated With Successful Gastrostomy Tube Weaning in Patients With Prolonged Dysphagia After Stroke
}

\author{
Bo Seong Jang, MD, Jun Young Park, MD, Jae Hyun Lee, MD, Young Joo Sim, MD, PhD, \\ Ho Joong Jeong, MD, PhD, Ghi Chan Kim, MD, PhD
}

Department of Physical Medicine and Rehabilitation, Kosin University College of Medicine, Busan, Korea

\begin{abstract}
Objective To investigate the clinical factors associated with successful gastrostomy tube weaning in patients with prolonged dysphagia after stroke.

Methods This study involved a retrospective medical chart review of patients diagnosed with prolonged dysphagia after stroke who underwent gastrostomy tube insertion between May 2013 and January 2020. Forty-seven patients were enrolled and consequently divided into gastrostomy tube sustaining and weaning groups. The numbers of patients in the sustaining and weaning groups were 31 and 16, respectively. The patients' demographic data, Korean version of Mini-Mental State Examination (K-MMSE) score, Korean version of the Modified Barthel Index (K-MBI), Functional Dysphagia Scale (FDS) score, and Penetration-Aspiration Scale (PAS) score were compared between the two groups. A videofluoroscopic swallowing study was performed before making the decision of gastrostomy tube weaning. The clinical factors associated with gastrostomy tube weaning were then investigated. Results There were significant differences in age; history of aspiration pneumonia; K-MMSE, FDS, and PAS scores; and K-MBI between the groups. In the multiple logistic regression analysis, the FDS (odds ratio [OR]=0.791; 95\% confidence interval [CI], 0.634-0.987) and PAS scores (OR=0.205; 95\% CI, 0.059-0.718) were associated with successful gastrostomy tube weaning. In the receiver operating characteristic curve analysis, the FDS and PAS were useful screening tools for successful weaning, with areas under the curve of 0.911 and 0.918 , respectively.

Conclusion In patients with prolonged dysphagia, the FDS and PAS scores are the only factors associated with successful gastrostomy tube weaning. An evaluation of the swallowing function is necessary before deciding to initiate gastrostomy tube weaning.
\end{abstract}

Keywords Dysphagia, Stroke, Gastrostomy

Received July 21, 2020; Revised August 24, 2020; Accepted September 23, 2020; Published online February 9, 2021

Corresponding author: Ghi Chan Kim

Department of Physical Medicine and Rehabilitation, Kosin University College of Medicine, 262 Gamcheon-ro, Seo-gu, Busan 49267, Korea. Tel: +8251-990-6156, Fax: +82-51-990-3181, E-mail: ghichan@hotmail.com

ORCID: Bo Seong Jang (https://orcid.org/0000-0003-2109-2226); Jun Young Park (https://orcid.org/0000-0002-5443-4416); Jae Hyun Lee (https:// orcid.org/0000-0002-4927-9722); Young Joo Sim (https://orcid.org/0000-0002-0640-8766); Ho Joong Jeong (https://orcid.org/0000-0002-0607-2799); Ghi Chan Kim (https://orcid.org/0000-0002-4954-3175).

(c) This is an open-access article distributed under the terms of the Creative Commons Attribution Non-Commercial License (http://creativecommons.org/ licenses/by-nc/4.0) which permits unrestricted noncommercial use, distribution, and reproduction in any medium, provided the original work is properly cited. Copyright $\odot 2021$ by Korean Academy of Rehabilitation Medicine 


\section{INTRODUCTION}

Dysphagia is a common complication after stroke, causing a nutritional deficit and aspiration pneumonia. In patients with dysphagia after stroke, enteral feeding through a tube is essential when they find it difficult to feed orally. Recovery of the swallowing function is typically observed within 2 weeks; however, in $11 \%-13 \%$ of patients with stroke, dysphagia can persist for months [1]. Continuous feeding through a nasogastric tube in patients with prolonged dysphagia induces complications, such as aspiration pneumonia, vocal cord dysfunction, and gastroesophageal reflux [2]. Therefore, percutaneous gastrostomy is recommended in patients with prolonged dysphagia when swallowing difficulty persists for more than 3 weeks [3].

In a previous study, feeding through a gastrostomy tube during long-term rehabilitation was found to be safer and more effective than feeding through a nasogastric tube [4]. In another study, feeding through a gastrostomy tube resulted in improved nutritional status and fewer complications, including aspiration pneumonia, than did feeding through a nasogastric tube, in patients with dysphagia lasting more than 6 weeks [5].

However, sustained feeding through a gastrostomy tube causes complications, such as granuloma formation and local wound infection, which can be an obstacle to active rehabilitation [3]. Therefore, gastrostomy tube weaning is advisable when continuous neurorehabilitation improves the swallowing function and makes oral feeding possible. Several studies have been conducted on the clinical factors associated with gastrostomy tube weaning based on videofluoroscopic swallowing study (VFSS) findings. One study suggested that the absence of aspiration and absence of pharyngeal trigger delay were predictive factors for percutaneous endoscopic gastrostomy (PEG) tube removal [6]. Another study suggested that aspiration during VFSS was a predictor of gastrostomy tube removal [7]. However, screening tests for gastrostomy tube weaning have not been reported. In addition, most previous studies did not apply scales commonly used in the interpretation of the VFSS findings. In other studies, the scales used to interpret the VFSS findings were different. Although rare, some patients who were successfully weaned from a gastrostomy tube had an enteral feeding tube inserted owing to various causes in clinical trials. However, knowl- edge on these causes is limited.

Herein, we investigated the clinical factors and screening tests associated with successful gastrostomy tube weaning in patients with prolonged dysphagia after stroke. The VFSS findings were interpreted using scales commonly applied in clinical settings. Further, we investigated the cause for reinsertion of enteral feeding tubes among patients successfully weaned from a gastrostomy tube.

\section{MATERIALS AND METHODS}

\section{Subjects}

This study involved a retrospective review of the medical charts of patients diagnosed with acute stroke at Kosin University Gospel Hospital between May 2013 and January 2020. It was approved by the Institutional Review Board of Kosin University Gospel Hospital (No. 2020-07022). The informed consent was waived. The inclusion criteria were (1) a diagnosis of stroke following a neurological examination and brain imaging; (2) obtainment of data from VFSS before deciding to wean the patient from a gastrostomy tube; (3) PEG or percutaneous radiologic gastrostomy (PRG); and (4) prolonged dysphagia requiring non-oral feeding for 2 weeks or more after onset of acute stroke. Conversely, the exclusion criteria were (1) a history of stroke; (2) unexpected gastrostomy tube removal owing to dislodgement; (3) reinsertion of an enteral feeding tube after gastrostomy tube weaning; and (4) multifocal or bilateral lesions of the brain.

According to previous studies, gastrostomy tube insertion is usually recommended when patients with stroke need long-term enteral feeding for more than 4 weeks [24]. Gastrostomy tube insertion was determined on the basis of the findings of the initial VFSS performed during acute stroke and follow-up VFSS performed after 3 weeks of stroke onset. Based on the findings of the latter, we determined who among the patients needed long-term enteral feeding for more than 4 weeks. Among these patients, gastrostomy tube insertion was performed when no recovery of dysphagia was observed in the follow-up VFSS compared with the initial VFSS and when aspiration was observed.

A total of 1,023 patients were screened during the retrospective study. Among these patients, 92 underwent gastrostomy tube insertion owing to prolonged dyspha- 
gia, and all of them underwent VFSS. Thirty-five patients were excluded in accordance with the exclusion criteria; seven patients were further excluded because swallowing rehabilitation was discontinued owing to worsening of their medical condition. Among the patients whose stroke onset exceeded 1 month, there were three cases of nasogastric tube to gastrostomy tube conversion. These patients were also excluded because the mean duration from stroke onset to gastrostomy tube insertion differed from that in this study. Finally, 47 patients were enrolled, with 31 patients $(66 \%)$ categorized in a sustaining group and 16 (34\%) in a weaning group.

Using medical records, we investigated the patients' sex, age, type of stroke, location of the brain lesion, lesion hemisphere, type of percutaneous gastrostomy, history of aspiration pneumonia, functional status, and VFSS findings. The type of stroke was divided into ischemic and hemorrhagic types. Subarachnoid hemorrhages were excluded. The location of the brain lesion was divided into brainstem and non-brainstem locations. Lesions in the middle, anterior, and posterior cerebral arteries were assigned to the non-brainstem group and lesions in the midbrain, pons, and medulla to the brainstem group. The lesion hemispheres were divided into left and right hemispheres. The gastrostomy type was divided into PEG and PRG. Functional status was determined using the Korean version of the Mini-Mental State Examination (KMMSE) and the Korean version of the Modified Barthel Index (K-MBI); the K-MMSE was used to measure the cognitive function and the K-MBI to measure activities of daily living. The K-MMSE and K-MBI were used before making the decision of gastrostomy tube weaning.

All patients underwent appropriate rehabilitation for swallowing difficulty until the decision on gastrostomy tube weaning. After the VFSS, the patients were classified into the sustaining group or weaning group. The decision of gastrostomy tube weaning was achieved through an iterative group discussion involving the attending physician, occupational therapist, and experienced physician with expertise on dysphagia. Gastrostomy tube weaning was determined on the basis of (1) recovery of swallowing difficulty following dysphagia rehabilitation, (2) ability to maintain a sitting position, and (3) whether aspiration disappears in VFSS when a food thickener is used. After gastrostomy tube weaning, all patients were fed a dysphagia diet. Over the course of 3 days, the women aimed at consuming 1,800 $\mathrm{kcal} /$ day and men at consuming $2,100 \mathrm{kcal} /$ day. All patients were transferred to another hospital after weaning or sustaining the gastrostomy tube and followed up for 3 months as outpatients. Among the patients weaned off from a gastrostomy tube, we investigated those who underwent reinsertion of an enteral feeding tube and the cause of reinsertion. These patients were then excluded from the weaning group because of the reinsertion of an enteral feeding tube.

\section{VFSS}

In this study, all participants underwent gastrostomy tube insertion 3 weeks after the onset of acute stroke. At approximately 3 months after tube insertion, VFSS was performed by an experienced radiology technician before the decision on gastrostomy tube weaning. The participants were asked to swallow a barium-impregnated bolus comprising $5 \mathrm{~mL}$ of a thin liquid and pudding. The VFSS was recorded using video clips, and two rehabilitation physicians interpreted the clips. The VFSS findings were interpreted using the Functional Dysphagia Scale (FDS) and the Penetration-Aspiration Scale (PAS) $[8,9]$. If the interpretations differed between the two rehabilitation physicians, we adopted the interpretation of the more experienced physician.

Both the FDS and PAS are commonly used to interpret VFSS findings in clinical settings. The FDS consists of the oral and pharyngeal phases, with possible scores of 0 to a maximum of 100 points. The closer the FDS score is to 100 points, the greater the severity of swallowing difficulty. Conversely, the PAS consists of the presence or absence of penetration, aspiration, level of airway invasion, and ability for ejection after penetration or aspiration, with a minimum score of 1 point to a maximum of 8 points. The closer the PAS score is to 8 points, the greater the severity of swallowing difficulty.

\section{Statistical analysis}

The Mann-Whitney U test and Pearson chi-square test were performed to compare sex, age, the type of stroke, the location of the brain lesion, the type of gastrostomy, history of aspiration pneumonia, the K-MBI, and the KMMSE, FDS, and PAS scores between the sustaining and weaning groups. The components of the FDS between the sustaining and weaning groups were also compared. A multiple logistic regression analysis was used to inves- 
tigate the independent clinical factors associated with successful gastrostomy tube weaning in terms of odds ratios (OR) and 95\% confidence intervals (CIs), with the significance level set at $\mathrm{p}<0.05$. A receiver operating characteristic (ROC) curve was used to determine the screening tests associated with successful gastrostomy tube weaning, with the area under the curve (AUC) set at $>0.5$.
The cutoff value, sensitivity, and specificity of the screening tests were calculated using the ROC curve. A partial correlation analysis between the FDS and PAS was performed after adjusting for age and a reliability analysis to evaluate their inter-rater reliability. Statistical Package for the Social Sciences version 25.0 (IBM, Armonk, NY, USA) was used for statistical analysis.

Table 1. Demographic characteristics of the patients

\begin{tabular}{|c|c|c|c|}
\hline Variable & Sustaining group $(n=31)$ & Weaning group $(n=16)$ & p-value \\
\hline \multicolumn{4}{|l|}{ Sex } \\
\hline Male & $14(45)$ & $7(43)$ & 0.683 \\
\hline Female & $17(55)$ & $9(57)$ & \\
\hline Age (yr) & $72.5 \pm 6.8$ & $66.8 \pm 5.7$ & $0.012^{*}$ \\
\hline \multicolumn{4}{|l|}{ Duration (day) } \\
\hline From stroke onset to tube insertion & $21.8 \pm 3.3$ & $20.9 \pm 3.7$ & 0.289 \\
\hline From stroke onset to VFSS & $110.4 \pm 9.9$ & $108.9 \pm 12.2$ & 0.840 \\
\hline From tube insertion to weaning & & $90.9 \pm 10.8$ & \\
\hline \multicolumn{4}{|l|}{ Medical history } \\
\hline Hypertension & $18(58)$ & $8(50)$ & 0.598 \\
\hline Diabetes mellitus & $10(32)$ & $5(31)$ & 0.944 \\
\hline Coronary artery disease & $10(32)$ & $7(44)$ & 0.437 \\
\hline Atrial fibrillation & $9(29)$ & $6(38)$ & 0.555 \\
\hline Tracheostomy & $3(10)$ & $1(6)$ & 0.690 \\
\hline \multicolumn{4}{|l|}{ Type of stroke } \\
\hline Ischemic & $25(81)$ & $12(75)$ & 0.913 \\
\hline Hemorrhagic & $6(19)$ & $4(25)$ & \\
\hline \multicolumn{4}{|l|}{ Location of the brain lesion } \\
\hline Brainstem & $7(23)$ & $4(25)$ & 0.748 \\
\hline Non-brainstem & $24(77)$ & $12(75)$ & \\
\hline \multicolumn{4}{|l|}{ Lesion hemisphere } \\
\hline Left hemisphere & $28(90)$ & $13(81)$ & 0.238 \\
\hline Right hemisphere & $3(10)$ & $3(19)$ & \\
\hline \multicolumn{4}{|l|}{ Type of gastrostomy } \\
\hline PEG & $19(61)$ & $8(50)$ & 0.700 \\
\hline PRG & $12(39)$ & $8(50)$ & \\
\hline History of aspiration pneumonia & $14(45)$ & $2(12)$ & $0.045^{*}$ \\
\hline K-MBI & $34.8 \pm 9.8$ & $50.9 \pm 10.0$ & $<0.001^{* * *}$ \\
\hline K-MMSE score & $11.1 \pm 5.0$ & $15.9 \pm 5.4$ & $0.009^{* *}$ \\
\hline FDS score & $50.5 \pm 15.3$ & $25.4 \pm 8.4$ & $<0.001^{* * *}$ \\
\hline PAS score & $4.5 \pm 1.2$ & $2.2 \pm 1.0$ & $<0.001^{* * *}$ \\
\hline
\end{tabular}

Values are presented as number (\%) or mean \pm standard deviation.

VFSS, videofluoroscopic swallowing study; PEG, percutaneous endoscopic gastrostomy; PRG, percutaneous radiologic gastrostomy; K-MBI, Korean version of the Modified Barthel Index; K-MMSE, Korean version of the Mini-Mental State Examination; FDS, Functional Dysphagia Scale; PAS, Penetration-Aspiration Scale.

${ }^{*} \mathrm{p}<0.05,{ }^{* *} \mathrm{p}<0.01,{ }^{* * *} \mathrm{p}<0.001$ using the Mann-Whitney $\mathrm{U}$ test and Pearson chi-square test. 
Table 2. Comparison of the components of the FDS between the sustaining and weaning groups

\begin{tabular}{lccc}
\hline \multicolumn{1}{c}{ Component } & Sustaining group (n=31) & Weaning group (n=16) & p-value \\
\hline Lip closure & $18(58)$ & $7(44)$ & 0.351 \\
Bolus formation & $21(68)$ & $6(38)$ & 0.065 \\
Residue in the oral cavity & $17(55)$ & $8(50)$ & 0.753 \\
Oral transit time & $19(61)$ & $6(38)$ & 0.121 \\
Triggering of pharyngeal swallowing & $27(87)$ & $8(50)$ & $0.006^{* *}$ \\
Laryngeal elevation and epiglottic closure & $22(71)$ & $4(25)$ & $0.003^{* *}$ \\
Nasal penetration & $6(19)$ & $3(19)$ & 0.960 \\
Residue in the valleculae & $20(65)$ & $7(44)$ & 0.172 \\
Residue in the pyriform sinuses & $20(65)$ & $6(38)$ & 0.078 \\
Coating of the pharyngeal wall after swallowing & $23(74)$ & $6(38)$ & $0.014^{*}$ \\
Pharyngeal transit time & $23(74)$ & $9(56)$ & 0.211 \\
\hline
\end{tabular}

Values are presented as numbers (\%) of abnormal findings on videofluoroscopic swallowing study.

FDS, Functional Dysphagia Scale.

${ }^{*} \mathrm{p}<0.05,{ }^{* *} \mathrm{p}<0.01$ using Pearson chi-square test.

Table 3. Multiple logistic regression analysis of the independent clinical factors associated with successful gastrostomy tube weaning

\begin{tabular}{lcc}
\hline & OR (95\% CI) & p-value \\
\hline FDS score & $0.791(0.634-0.987)$ & $0.038^{*}$ \\
PAS score & $0.205(0.059-0.718)$ & $0.013^{*}$ \\
Age & $0.880(0.741-1.044)$ & 0.134 \\
$\begin{array}{l}\text { History of aspiration } \\
\text { pneumonia }\end{array}$ & $0.224(0.020-2.433)$ & 0.849 \\
K-MBI & & \\
K-MMSE score & $1.110(0.899-1.233)$ & 0.241 \\
\hline
\end{tabular}

OR, odds ratio; CI, confidence interval; FDS, Functional Dysphagia Scale; PAS, Penetration-Aspiration Scale; KMBI, Korean version of the Modified Barthel Index; KMMSE, Korean version of the Mini-Mental State Examination.

${ }^{*} \mathrm{p}<0.05$.

\section{RESULTS}

The demographic characteristics of the sustaining and weaning groups are presented in Table 1 . The mean duration from stroke onset to gastrostomy tube insertion was 21.8 and 20.9 days in the sustaining and weaning groups, respectively. The mean duration from stroke onset to VFSS was 110.4 and 108.9 days in the sustaining and weaning groups, respectively. The mean duration from gastrostomy tube insertion to weaning was 90.9 days in the weaning group. There were significant differences in

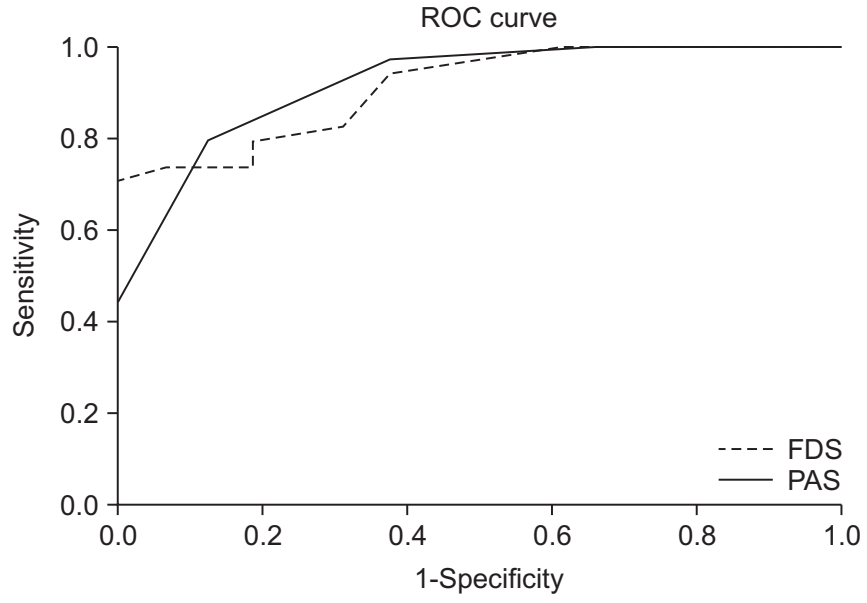

Fig. 1. Receiver operating characteristic (ROC) curve of the Functional Dysphagia Scale (FDS) and PenetrationAspiration Scale (PAS) for successful gastrostomy tube weaning.

age, history of aspiration pneumonia, the K-MBI, and the K-MMSE, FDS, and PAS scores between the two groups. However, there were no significant differences in sex and the type of stroke, location of the brain lesion, lesion hemisphere, and type of gastrostomy between the sustaining and weaning groups. In the comparison of the components of the FDS between the sustaining and weaning groups, significant differences in triggering of pharyngeal swallowing $(\mathrm{p}=0.006)$, laryngeal elevation and epiglottic closure $(\mathrm{p}=0.003)$, and coating of the pharyngeal wall after swallowing $(p=0.014)$ were observed 
(Table 2). In the multiple logistic regression analysis, the FDS (OR=0.791; 95\% CI, 0.634-0.987) and PAS scores ( $\mathrm{OR}=0.205 ; 95 \% \mathrm{CI}, 0.059-0.718$ ) were found to be the independent clinical factors associated with successful gastrostomy tube weaning (Table 3). In the ROC curve analysis, the FDS and PAS were found to be useful screening tests for successful gastrostomy tube weaning (Fig. 1). The FDS had an AUC of 0.911, with a sensitivity and specificity of $73.5 \%$ and $93.7 \%$, respectively, and a cutoff value of 37.5. Conversely, the PAS had an AUC of 0.918 , with a sensitivity and specificity of $79.4 \%$ and $87.5 \%$, respectively, and a cutoff value of 3.5 (Table 4). In the partial correlation analysis, there was a significant correlation found between the FDS and PAS after adjusting for age $(r=0.779$, $\mathrm{p}<0.001$ ) (Table 5). In the reliability analysis, the interrater reliability of the FDS and PAS was 0.655 and 0.831 , respectively. The causes for the reinsertion of an enteral feeding tube after gastrostomy tube weaning were aspiration pneumonia (33\%), recurrent stroke (33\%), myocardial infarction (22\%), and unknown cause (11\%) (Fig. 2).

\section{DISCUSSION}

The purpose of our study was to investigate the clinical factors and screening tests associated with successful gastrostomy tube weaning in patients with prolonged dysphagia after stroke. Currently, there is no existing gold standard for weaning gastrostomy tubes. This study is relevant in that the FDS and PAS were presented as screening tests for gastrostomy tube weaning, and the cutoff scores were reported. In addition, only a few previous studies have comprehensively considered the KMBI; FDS, PAS, and K-MMSE scores; demographic data in relation to the brain lesions; and gastrostomy type. In this study, the FDS and PAS scores were the only clinical factors associated with successful gastrostomy tube weaning.

In patients with swallowing difficulty after stroke, VFSS has been considered the gold standard for the diagnosis of dysphagia [10]. The methods used to interpret VFSS findings were different in each previous study. In this study, we interpreted the VFSS findings using the FDS and PAS, both of which are commonly used in clinical settings. Han et al. [11] suggested that the Videofluoroscopic Dysphagia Scale (VDS) can be useful in predicting persistent dysphagia in patients with stroke for more than 6 months. The components of the FDS are similar to those of the VDS. Similar to the previous study, our study showed that the FDS score served as an independent predictive clinical factor in patients with prolonged dysphagia. In comparison, the components of the FDS, i.e., triggering of pharyngeal swallowing, laryngeal elevation and epiglottic closure, and coating of the pharyngeal wall after swallowing, were significantly different between the sustaining and weaning groups in this study. A previous study has also suggested the absence of pharyngeal trigger delay and aspiration as predictive factors for the removal of PEG tubes [6].

The PAS offers the advantage that it takes relatively less time to interpret the scores than other methods and that it exhibits a high inter-rater reliability [12]. However, previous studies on the PAS were mostly based on nasogastric tube removal, and it was difficult to find a study of gastrostomy tube weaning based on the PAS score $[13,14]$. In this study, the PAS score was found to be an inde-

Table 4. ROC curve analysis of the AUC, cutoff values, sensitivity, and specificity of the FDS and PAS

\begin{tabular}{ccccc}
\hline Variable & AUC & Cutoff value & Sensitivity & Specificity \\
\hline FDS & 0.911 & 37.5 & 0.735 & 0.937 \\
PAS & 0.918 & 3.5 & 0.794 & 0.875 \\
\hline
\end{tabular}

ROC, receiver operating characteristic; AUC, area under the curve; FDS, Functional Dysphagia Scale; PAS, Penetration-Aspiration Scale.

Table 5. Correlation between the FDS and PAS

\begin{tabular}{ccc}
\hline & FDS & p-value \\
\hline PAS & 0.779 & $<0.001^{* * *}$ \\
\hline
\end{tabular}

The variable is the correlation coefficient.

FDS, Functional Dysphagia Scale; PAS, Penetration-Aspiration Scale.

${ }^{* * *} \mathrm{p}<0.001$ in the partial correlation analysis after adjusting for age.

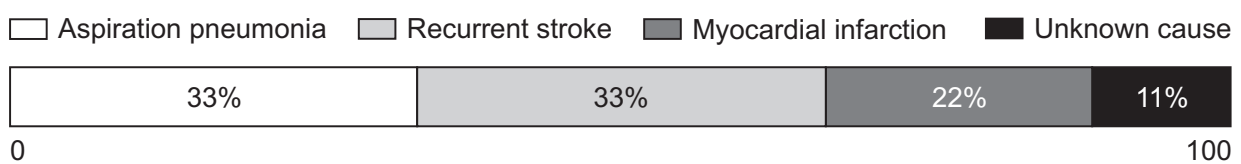


pendent predictive factor, and the PAS was considered a screening test for successful gastrostomy tube weaning. The FDS and PAS would then likely be useful for interpreting VFSS findings before planning gastrostomy tube weaning in patients with prolonged dysphagia after stroke.

In this study, the K-MBI and K-MMSE score were used to estimate the patients' functional status. A previous study has investigated the factors associated with PEG tube removal using the MBI and MMSE score [6]. Neither were predictive factors for the removal of the PEG tube in post-stroke dysphagia cases. In our study, the K-MBI and K-MMSE score were not found to be predictive independent factors for gastrostomy tube weaning. According to a previous study, prolonged dysphagia was significantly associated with the Barthel index [15]. The MBI, particularly for patients with under 20 points, was a useful component of assessment to predict prolonged dysphagia. The average K-MBI in the sustaining group in our study was 34.8 points, and most patients had a K-MBI higher than 20 points. Therefore, the K-MBI was not associated with successful gastrostomy tube weaning herein.

In some previous studies, there was no significant association found between the location of the brain lesion and prolonged dysphagia $[16,17]$. Our study found no significant differences in the location of the brain lesions (brainstem or non-brainstem) between the groups. Sex, brainstem infarct, right hemispheric infarct, and pneumonia also had no significant association with prolonged dysphagia. The relationship between the type of stroke and prolonged dysphagia is not well understood. According to a systematic review of dysphagia after stroke, there was no sufficient evidence to prove the effect of the type of stroke [18]. No significant difference in the type of stroke between the gastrostomy tube sustaining and weaning groups has been reported, as is the case in our study $[6,7]$.

In our study, there were no significant differences in the type of gastrostomy between the sustaining and weaning groups. In a previous study, PRG was superior to PEG in patients with amyotrophic lateral sclerosis [19]. However, we could not confirm the superiority of PRG because the cause of dysphagia differed from that in our study. In another study, PEG and PRG were found to be safe and useful for nutritional delivery in an acute care setting [20]. No significant difference in minor complications was found between the two methods, although a difference in cost was evident, with PEG costing $44 \%$ more than PRG [20]. In this study, no definite indication was seen with respect to the choice of PEG or PRG, and the type of gastrostomy was determined by the attending physician. In general, we observed no sufficient evidence for the superiority of gastrostomy-type selection.

In previous studies, age was reported as an independent predictive factor for gastrostomy tube weaning $[7,21]$. However, in our study, age was not found to be a predictive factor for gastrostomy tube weaning. This could be because younger patients with gastrostomy tubes had more severe stroke and irreversible dysphagia [6]. However, there were no data on the severity of stroke in our study. Therefore, further studies including the severity of stroke are needed.

We followed up patients in whom gastrostomy tubes were removed 3 months after weaning. Among the 25 patients who were weaned from a tube, nine experienced reinsertion of an enteral feeding device in the form of a nasogastric, PEG, or PRG tube. Laryngeal elevation and epiglottic closure were significantly different between the sustaining and reinsertion groups. When considering gastrostomy tube weaning, a careful evaluation of laryngeal elevation and epiglottic closure can be helpful in predicting reinsertion. The reasons for reinsertion were recurrent stroke, aspiration pneumonia, and myocardial infarction. These patients may not have fully recovered from dysphagia owing to aspiration pneumonia. Therefore, it is important to follow up patients weaned from a gastrostomy tube through continuous and regular VFSS.

Our study had some limitations. First, we could not compare the superiority between the FDS and PAS owing to the lack of a gold standard for gastrostomy tube weaning. Second, the severity of stroke and functional status at admission were not included. Finally, the cutoff score for the PAS was too low. A PAS score of 6 points or more indicates aspiration; however, the cutoff score for the PAS in this study was 3.5 points. It is thought that we strictly planned gastrostomy tube weaning.

A gastrostomy tube is an effective method of feeding a patient with prolonged dysphagia after stroke. However, long-term use of a gastrostomy tube can induce various complications, and oral feeding has various advantages compared with gastrostomy tube feeding. Therefore, gastrostomy tubes should be removed following recovery of 
the swallowing function. In our study, the FDS and PAS scores were the only factors associated with successful gastrostomy tube weaning. The FDS and PAS also proved to be useful screening tests for successful gastrostomy tube weaning. Therefore, it is important to evaluate the swallowing function using the FDS and PAS before making a decision on gastrostomy tube weaning in patients with prolonged dysphagia after stroke.

\section{CONFLICT OF INTEREST}

No potential conflict of interest relevant to this article was reported.

\section{AUTHOR CONTRIBUTION}

Conceptualization: Jang BS, Kim GC. Methodology: Jeong HJ, Sim YJ. Formal analysis: Jang BS, Park JY. Project administration: Kim GC, Lee JH. Visualization: Jang BS, Park JY. Writing of the original draft: Jang BS. Writing, reviewing, and editing: Jang BS, Kim GC, Lee JH. Approval of the final manuscript: all authors.

\section{REFERENCES}

1. Gonzalez-Fernandez M, Ottenstein L, Atanelov L, Christian AB. Dysphagia after stroke: an overview. Curr Phys Med Rehabil Rep 2013;1:187-96.

2. Qureshi AZ, Jenkins RM, Thornhill TH. Percutaneous endoscopic gastrostomy versus nasogastric tube feeding during neurorehabilitation. Ifs, ands, or buts. Neurosciences (Riyadh) 2016;21:69-71.

3. Rahnemai-Azar AA, Rahnemaiazar AA, Naghshizadian R, Kurtz A, Farkas DT. Percutaneous endoscopic gastrostomy: indications, technique, complications and management. World J Gastroenterol 2014;20:7739-51.

4. Park RH, Allison MC, Lang J, Spence E, Morris AJ, Danesh BJ, et al. Randomised comparison of percutaneous endoscopic gastrostomy and nasogastric tube feeding in patients with persisting neurological dysphagia. BMJ 1992;304:1406-9.

5. Kim CJ, Chun MH, Ha SB. Comparison of percutaneous endoscopic gastrostomy and nasogastric tube feeding in dysphagic stroke. J Korean Acad Rehabil Med 1997;21:1110-7.
6. Yi Y, Yang EJ, Kim J, Kim WJ, Min Y, Paik NJ. Predictive factors for removal of percutaneous endoscopic gastrostomy tube in post-stroke dysphagia. J Rehabil Med 2012;44:922-5.

7. Ickenstein GW, Kelly PJ, Furie KL, Ambrosi D, Rallis N, Goldstein R, et al. Predictors of feeding gastrostomy tube removal in stroke patients with dysphagia. J Stroke Cerebrovasc Dis 2003;12:169-74.

8. Han TR, Paik NJ, Park JW. Quantifying swallowing function after stroke: a functional dysphagia scale based on videofluoroscopic studies. Arch Phys Med Rehabil 2001;82:677-82.

9. Rosenbek JC, Robbins JA, Roecker EB, Coyle JL, Wood JL. A penetration-aspiration scale. Dysphagia 1996;11:93-8.

10. Cohen DL, Roffe C, Beavan J, Blackett B, Fairfield CA, Hamdy S, et al. Post-stroke dysphagia: a review and design considerations for future trials. Int J Stroke 2016;11:399-411.

11. Han TR, Paik NJ, Park JW, Kwon BS. The prediction of persistent dysphagia beyond six months after stroke. Dysphagia 2008;23:59-64.

12. Butler SG, Markley L, Sanders B, Stuart A. Reliability of the penetration aspiration scale with flexible endoscopic evaluation of swallowing. Ann Otol Rhinol Laryngol 2015;124:480-3.

13. Lee JH, Kim SB, Lee KW, Lee SJ, Park JG, Ri JW. Associating factors regarding nasogastric tube removal in patients with Dysphagia after stroke. Ann Rehabil Med 2014;38:6-12.

14. Hwang JM, Cheong YS, Kang MG, Chun SM, Min YS, Lee YS, et al. Recommendation of nasogastric tube removal in acute stroke patients based on videofluoroscopic swallow study. Ann Rehabil Med 2017;41:915.

15. Broadley S, Croser D, Cottrell J, Creevy M, Teo E, Yiu D, et al. Predictors of prolonged dysphagia following acute stroke. J Clin Neurosci 2003;10:300-5.

16. Kumar S, Doughty C, Doros G, Selim M, Lahoti S, Gokhale S, et al. Recovery of swallowing after dysphagic stroke: an analysis of prognostic factors. J Stroke Cerebrovasc Dis 2014;23:56-62.

17. Daniels SK, Pathak S, Mukhi SV, Stach CB, Morgan RO, Anderson JA. The relationship between lesion localization and dysphagia in acute stroke. Dysphagia 2017;32:777-84. 
18. Martino R, Foley N, Bhogal S, Diamant N, Speechley M, Teasell R. Dysphagia after stroke: incidence, diagnosis, and pulmonary complications. Stroke 2005;36:2756-63.

19. Thornton FJ, Fotheringham T, Alexander M, Hardiman O, McGrath FP, Lee MJ. Amyotrophic lateral sclerosis: enteral nutrition provision. Endoscopic or radiologic gastrostomy? Radiology 2002;224:713-7.

20. Galaski A, Peng WW, Ellis M, Darling P, Common A, Tucker E. Gastrostomy tube placement by radiologi- cal versus endoscopic methods in an acute care setting: a retrospective review of frequency, indications, complications and outcomes. Can J Gastroenterol 2009;23:109-14.

21. Oto T, Kandori Y, Ohta T, Domen K, Koyama T. Predicting the chance of weaning dysphagic stroke patients from enteral nutrition: a multivariate logistic modelling study. Eur J Phys Rehabil Med 2009;45:35562. 